\title{
Aspectos Relacionados à Fadiga Durante o Ciclismo: Uma Abordagem Biomecânica
}

\section{Aspects Related With Fatigue During Cycling: a Biomechanical Approach}

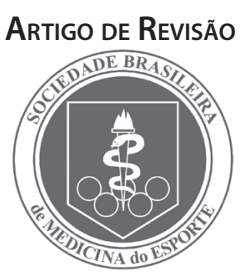

Fernando Diefenthaeler

Marco Aurélio Vaz

Escola de Educação Física

- Laboratório de Pesquisa do

Exercício

Universidade Federal do Rio Grande do Sul, Porto Alegre - RS

\section{Endereço para correspondência: Fernando Diefenthaeler \\ Escola de Educação Física \\ - Laboratório de Pesquisa do \\ Exercício, Universidade Federal do \\ Rio Grande do Sul \\ Rua Felizardo 750, LAPEX, sala 212. \\ CEP 90690-200 \\ Telefone: (51) 3308-5859 \\ E-mail: fdiefenthaeler@gmail.com}

Submetido em 19/11/2007

Versão final recebida em 13/03/2008

Aceito em 04/04/2008

\begin{abstract}
RESUMO
A fadiga muscular pode ser definida como a incapacidade funcional na manutenção de um nível esperado de força. As competições de ciclismo, especialmente provas de estrada, apresentam como característica longa duração e altas intensidades. Tais características resultam na instauração do processo de fadiga, que pode estar associado a mecanismos e fatores metabólicos que afetam os músculos (fadiga periférica) e o sistema nervoso central (fadiga central). O objetivo deste trabalho é fazer uma revisão sobre aspectos relacionados com as mudanças na técnica de pedalada e na atividade elétrica dos músculos envolvidos nesse movimento durante o processo de fadiga. Alguns desses aspectos têm sido reportados na literatura e podem ter repercussão na (1) magnitude, direção e sentido de aplicação das forças no pedal; no (2) padrão de ativação muscular; na (3) geração de força e, conseqüentemente, no (4) desempenho do ciclista. No entanto, poucos estudos associam a fadiga muscular ao comportamento das forças aplicadas no pedal e ao padrão da ativação muscular. Os resultados dos estudos revisados demonstram a incapacidade dos ciclistas em manter a força desejada, perda da técnica de pedalada e mudança nos padrões de ativação elétrica sob condições de fadiga.
\end{abstract}

Palavras-chave: fadiga, biomecânica, desempenho, ciclismo.

\begin{abstract}
Muscular fatigue can be defined as functional inability to maintain a desired force output. During cycling competition, especially road races, cyclists are required to exercise for extended duration at high intensities. These features often result in fatigue, which can be associated with metabolic mechanisms and factors affecting both muscles (peripheral fatigue) and the central nervous system (central fatigue). The aim of this study is to review aspects related to alterations in the pedaling technique and electrical activation of the muscles during a fatiguing exercise bout. Some of these alterations have been reported in the literature and can reflect on the (1) magnitude, direction, and sign of the forces applied on the pedal; (2) muscle activation pattern; (3) force generation; (4) and consequent performance of the cyclist. However, few studies have associated fatigue with pedal forces and muscle activation. The results of the reviewed studies have shown that cyclists failed to maintain a preset force level, alteration in the pedaling technique, and changes on the muscle activation pattern during cycling under fatigue condition.
\end{abstract}

Keywords: fatigue, biomechanics, performance, cycling.

\section{INTRODUÇÃO}

O ciclismo é considerado um esporte complexo. As competições são divididas em provas de pista (velódromo) e provas de rua (estrada), individualmente ou por equipes ${ }^{(1,2)}$. As provas de estrada apresentam características distintas em relação à duração, intensidade e topografia. Entre elas podemos destacar o Tour de France, com um percurso de aproximadamente $3.000 \mathrm{~km}$, percorridos durante 21 dias ininterruptos, entre os mais variados tipos de terrenos ${ }^{(3)}$.

As competições de estrada têm como característica a manutenção de alta intensidade por tempo prolongado, o que resulta na instauração do processo de fadiga, principalmente em função das mudanças na produção de torques resultantes das contrações musculares e na atividade elétrica dos músculos dos membros inferiores ${ }^{(2,4-6)}$.

A fadiga muscular pode ser definida como um conjunto de alterações decorrentes do trabalho ou exercício prolongado, gerando incapacidade funcional na manutenção de um nível esperado de for$c^{c^{(7,8)}}$. Dessa forma, a fadiga muscular está associada a mecanismos e fatores metabólicos, que podem afetar os músculos (fadiga periférica) e o sistema nervoso central-SNC (fadiga central) durante a realização de exercício intenso em atletas ${ }^{(9,10)}$

Há evidências a favor e contra o fato de o SNC constituir um sítio de fadiga. Todavia, alguns estudos apontam a periferia (ou o músculo esquelético mais especificamente) como o principal sítio da fadiga, pois fatores metabólicos que impedem a produção de força muscular têm sido observados ${ }^{(11,12)}$.

As alterações metabólicas no ciclismo, como a depleção de substratos de energia e o acúmulo de derivados metabólicos, são dependentes da intensidade e duração da prova. Em atividades com intensidade entre 60 e $90 \%$ do consumo máximo de oxigênio $\left(\mathrm{VO}_{2 \mathrm{MAX}}\right)$, a fadiga ocorre em decorrência da depleção das reservas de glicogênio, en- 
quanto que, em intensidades acima de $90 \%$ do $\mathrm{VO}_{2 \text { MAx }}$ a redução do desempenho acontece em função da produção excessiva de lactato sangüíneo ${ }^{(13)}$.

Como conseqüência das modificações metabólicas causadas pela fadiga durante a pedalada, podemos apontar as mudanças nos padrões de ativação muscular e na capacidade de produção de força ${ }^{(4,14,15)}$. Tais alterações têm sido descritas na literatura e podem ter repercussão na magnitude, direção e sentido de aplicação das forças no pedal, afetando assim a técnica da pedalada e, conseqüentemente, o desempenho do atleta.

Além das alterações metabólicas, a fadiga periférica pode também ser dependente de fatores centrais, sendo associada, por exemplo, a falhas na transmissão do estímulo na junção neuromuscular, falhas na ativação do sarcolema, falha na condução do potencial de ação nos túbulos transversos ou falha na liberação de cálcio pelo retículo sarcoplasmático ${ }^{(16)}$.

Em um estudo envolvendo ciclistas treinados, foi observada redução na produção de torque dos músculos extensores da articulação do joelho, logo após o término de uma prova de estrada ${ }^{(6)}$. De acordo com os autores, a incapacidade na geração de força não ocorreu devido à fadiga central, mas sim à fadiga periférica (fadiga muscular), pois, mesmo após percorrer 140km, os ciclistas avaliados apresentaram aumento nos picos de ativação elétrica durante um teste de contração voluntária máxima (CVM).

No entanto, Lepers et al.(4) analisaram os efeitos da fadiga muscular no torque e na atividade elétrica de nove ciclistas treinados. Os atletas pedalaram durante cinco horas a 55\% da potência aeróbica máxima. Os autores verificaram redução de 18\% no torque durante testes de CVM (pré e pós) e decréscimo na atividade elétrica dos músculos vastus lateralis (VL) e vastus medialis (VM) a partir da primeira hora de exercício, o que significa que houve fadiga tanto do SNC quanto periférica.

A presença de fadiga muscular, seja de natureza central, periférica ou ambas, possui ampla repercussão no ciclismo, uma vez que as modificações que ocorrem na condução do estímulo nervoso e na placa motora provocam prejuízos no desempenho durante competições, particularmente em provas de longa distância.

A partir da literatura revisada, que compreendeu o período de 1977 a 2007, é possível constatar que o processo de fadiga pode apresentar origens distintas e provocar a redução do desempenho dos ciclistas. Entretanto, existe uma lacuna no que diz respeito a estudos que associem a fadiga muscular com o comportamento das forças aplicadas no pedal e o padrão da ativação muscular no ciclismo. Sendo assim, o presente estudo tem como objetivo principal abordar as modificações na técnica e na atividade elétrica dos músculos envolvidos no movimento da pedalada em resposta à instalação dos mecanismos de fadiga.

\section{EFEITOS DA FADIGA SOBRE AS FORÇAS APLICADAS NO PEDAL}

\section{Aspectos relacionados com a dinamometria}

O estudo do movimento que inclui a análise da força como causa do movimento é chamada de cinética, mensurada por meio de uma técnica denominada de dinamometria ${ }^{(17)}$. A dinamometria tem sido amplamente utilizada para analisar a fadiga durante atividades físicas. O uso de células de carga e dinamômetros isocinéticos, por exemplo, tem possibilitado avaliar o índice de fadiga, calculado como a razão entre a força final e a força inicial, em treinamento de resistência(18).
O uso de pedais instrumentados tem possibilitado registrar as forças aplicadas pelo ciclista durante a pedalada com o objetivo de mensurar sua magnitude, sua orientação e sua técnica ${ }^{(19-23)}$. No entanto, os estudos envolvendo a análise das forças, na sua grande maioria, descrevem o padrão do movimento da pedalada na tentativa de identificar a técnica empregada pelo atleta, otimizando assim a energia despendida(24-27).

A componente da força resultante é obtida mediante combinação das componentes normal e tangencial aplicadas no pedal, e representa a força total, no plano sagital, aplicada pelo ciclista no pedal. Além das forças normal e tangencial, há também outras duas forças envolvidas no movimento da pedalada: (1) a força efetiva, que é a componente da força aplicada perpendicularmente ao pé-de-vela e que produz torque positivo, também chamada de força transmitida; e (2) a força inefetiva, que é a componente da força paralela ao pé-de-vela e que não produz torque ${ }^{(25,26,28,29)}$.

\section{Orientação e magnitude das forças}

A análise da aplicação das forças nos pedais durante a pedalada pode também ser um ótimo parâmetro para a descrição do processo de fadiga, e também elucidar quais estratégias os ciclistas utilizam para manter a mesma carga quando estão sob condições de fadiga ${ }^{(30-32)}$.

Para exemplificar o processo de fadiga durante uma prova de ciclismo, podemos apontar as mudanças na magnitude e na orientação das forças aplicadas no pedal, bem como o seu aproveitamento (força efetiva). Carpes et al. ${ }^{(33)}$, em um estudo preliminar, avaliaram o comportamento das forças aplicadas no pedal durante uma simulação de uma prova de $40 \mathrm{~km}$ contra-relógio em laboratório. Os resultados demonstraram aumento progressivo da força efetiva e diminuição da força inefetiva ao longo do teste, indicando melhora na técnica da pedalada.

Em contrapartida, o ciclista avaliado apresentou alteração na magnitude da força tangencial ao longo do teste. Essa modificação pode estar associada ao processo de fadiga, tendo em vista que o teste teve duração de aproximadamente 62 minutos em intensidade próxima à do limiar anaeróbio. Sendo assim, a melhora no aproveitamento das forças ocorreu em detrimento da habilidade do ciclista em "puxar" o pedal durante a fase de recuperação da pedalada.

O mesmo comportamento foi observado nas componentes de força normal e tangencial aplicadas no pedal em um estudo realizado por Amoroso et al. ${ }^{(31)}$. Os autores avaliaram 11 ciclistas de elite, em ciclossimulador, com uma carga de 300W. Os sujeitos foram encorajados a pedalar nessa intensidade durante o máximo tempo possível em que pudessem sustentar uma cadência de 80rpm (rotações por minuto).

Para avaliar os resultados, os autores dividiram o teste em fase inicial e final, denominando assim de período sem fadiga (NF) e com fadiga $(F)$, respectivamente. Os ciclistas avaliados apresentaram aumento no pico da força normal ( $N F=339,4 \mathrm{~N} ; \mathrm{F}=369,5 \mathrm{~N})$, e redução no pico de força tangencial (NF=79,1N; F=68,2N) durante a fase de propulsão da pedalada (0-180 ). No entanto, não houve diferença na magnitude e tampouco no comportamento das curvas de força resultante e efetiva, evidenciando que a técnica de pedalada não foi estratégia utilizada pelos ciclistas para superar a fadiga.

Ao avaliar o efeito da fadiga sobre as forças aplicadas no pedal de ciclistas treinados, Sanderson e Black ${ }^{(32)}$ também observaram aumento no pico da força normal. Os autores coletaram os dados dos pedais a cada minuto; os resultados apresentados eram referentes ao primeiro e ao último minuto (354 $\pm 65,0 \mathrm{~N}$ e $391 \pm 47,8 \mathrm{~N}$, respectivamente). 
No entanto, para força tangencial não houve diferença entre os dois instantes avaliados $(85,6 \pm 18,3 \mathrm{~N}$ e 85,3 \pm 19,4N).

Embora os resultados da força tangencial não tenham apresentado diferenças, o comportamento da curva, expressa em função do ângulo do pé-de-vela, apresentou mudanças. Os ciclistas adiantaram o ângulo de transição da fase de "puxar" o pedal para trás (de $133^{\circ}$ para 1210), e atrasaram a fase de transição de "empurrar" o pedal para frente (de $300^{\circ}$ para $330^{\circ}$ ), despendendo assim mais tempo aplicando força no pedal para trás.

Ao contrário do trabalho de Amoroso et al.(31), o estudo acima encontrou diferenças em relação à força efetiva. Os ciclistas apresentaram aumento no valor de pico da força efetiva nos dois instantes comparados ( $340 \pm 65,0 \mathrm{~N}$ e $377 \pm 74,8 \mathrm{~N}$, respectivamente). A partir desse resultado seria possível concluir que os ciclistas foram capazes de aproveitar mais as forças aplicadas no pedal no decorrer do teste, tornando-se mais técnicos. No entanto, também houve aumento da força inefetiva (componente de força que produz torque resistivo-negativo) de $-31,8 \pm 30,4 \mathrm{~N}$ para $-51,5 \pm 28,1 \mathrm{~N}$, respectivamente.

Segundo os autores, em função do processo de fadiga, os ciclistas tornaram-se menos efetivos durante a fase de recuperação (180-360\%), o que, em contrapartida, exigiu que os atletas aplicassem mais força durante a fase de propulsão. Talvez, em termos de gasto energético, essa troca não tenha gerado desequilíbrio, mas provavelmente os músculos extensores do joelho, responsáveis pela geração de força durante a fase de propulsão, foram sobrecarregados.

Em um estudo utilizando um cicloergômetro equipado com um sistema de pé-de-vela instrumentado com strain gauges (SRM ${ }^{\circ}$ powercontrol), Duc et al.(15) analisaram o comportamento da atividade elétrica de quatro músculos do membro inferior e o torque na fase de propulsão da pedalada, de ciclistas competitivos, durante um teste de 30 minutos. Os resultados não apontaram alterações no padrão das curvas de torque ao longo do teste.

Porém, a limitação do equipamento utilizado nesse estudo não permitiu avaliar o torque durante a fase de recuperação (sistema mede apenas o torque na fase de propulsão), nem as componentes da força normal e tangencial, restringindo assim a análise da técnica da pedalada empregada pelos ciclistas.

Utilizando um cicloergômetro SRM ${ }^{\circ}$ equipamento com o mesmo sistema SRM powercontrol já descrito, Carpes et al. ${ }^{(34)}$ avaliaram seis ciclistas treinados durante um teste de $40 \mathrm{~km}$ contra-relógio. Os sujeitos foram encorajados a realizar o teste no menor tempo possível, com cadência de pedalada livre. Os dados de torque foram coletados a cada cinco minutos até o termino do teste. Para análise, o teste foi dividido em quatro estágios de igual duração, aproximadamente, 15 minutos.

Os resultados demonstram aumento no pico do torque na fase de propulsão entre o primeiro e o quarto estágio $(15,6 \pm 6,5 \mathrm{~N} \cdot \mathrm{m}$ e 18,1 $\pm 4,6 \mathrm{~N} \cdot \mathrm{m}$, respectivamente). Paralelo às informações de torque, os autores coletaram o consumo de oxigênio $\left(\mathrm{VO}_{2}\right)$ ao longo do experimento. Essa variável também apresentou alterações entre o primeiro e o quarto estágio $\left(64,7 \pm 8,9 \% \mathrm{VO}_{2 \mathrm{PICO}}\right.$ e $\left.71,8 \pm 7,8 \% \mathrm{VO}_{2 \mathrm{PICO}}\right)$, o que provavelmente reflete a estratégia adotada pelos ciclistas para realizar o teste de forma progressiva.

Sendo assim, as mudanças observadas no pico de torque nesse estudo não representam melhora no aproveitamento das forças, mas possivelmente um reflexo do aumento do custo metabólico $\left(\mathrm{VO}_{2}\right)$ ao longo do teste.

\section{EFEITOS DA FADIGA NA ATIVIDADE ELÉTRICA DU- RANTE O CICLISMO}

\section{Aspectos relacionados com a eletromiografia}

A eletromiografia de superfície (EMG) fornece informações sobre o padrão de ativação muscular e sobre como o sistema nervoso central controla o movimento. Essa técnica tem sido amplamente utilizada para estudar a atividade muscular e a coordenação neuromuscular no ciclismo ${ }^{(26,35-40)}$.

A partir do monitoramento do padrão da atividade elétrica dos músculos envolvidos no movimento da pedalada, é possível obter informações sobre o comportamento do sistema neuromuscular. Durante atividades intensas e de longa duração, os músculos em processo de fadiga dão início a uma série de eventos que influenciam a eficiência e a capacidade de geração de força dos músculos ${ }^{(4,41,42)}$.

A análise de fadiga muscular tem sido proposta por meio do estudo da variação da amplitude do sinal eletromiográfico (geralmente medida pelo valor RMS - root mean square) em função do tempo. À proporção que ocorre aumento no número e no tamanho das unidades motoras recrutadas para a manutenção do nível de força desejado, há aumento do valor $\operatorname{RMS}^{(35,43)}$

Outra forma de avaliação do processo de fadiga pode ser realizada por meio da análise do sinal EMG através da transformada rápida de Fourier (FFT). A identificação de parâmetros do sinal EMG a partir da utilização da FFT, como a freqüência mediana do espectro de potência, pode evidenciar a relação entre altas e baixas freqüências no decorrer da atividade muscular ${ }^{(41)}$. Em contrações isométricas, ocorre um deslocamento para esquerda do espectro de potência, em direção às menores freqüências, com a fadiga ${ }^{(44)}$.

\section{Fadiga neuromuscular no ciclismo}

Muitos estudos relacionam a fadiga no ciclismo em provas de longa duração com fatores metabólicos (depleção das reservas de PCr, ATP, glicogênio, acúmulo de metabólicos, etc.); no entanto, pouco se sabe acerca da ocorrência da fadiga neuromuscular em eventos dessa natureza.

Na tentativa de elucidar os efeitos da fadiga no ciclismo prolongado sobre os parâmetros neuromusculares, metabólicos e biomecânicos, Lepers et al. ${ }^{(42)}$ avaliaram oito ciclistas treinados. Os atletas apresentaram aumento significativo no consumo de oxigênio $\left(\mathrm{VO}_{2}\right)$, na ventilação (VE) e na freqüência cardíaca (FC) ao longo das duas horas de avaliação. Em contrapartida, durante os testes (isométricos, concêntricos e excêntricos) realizados em um dinamômetro isocinético, antes e logo após o teste de ciclismo, foi observada redução no pico de torque e na atividade elétrica dos músculos VL e VM entre os dois momentos avaliados. Esses resultados indicam uma situação de fadiga periférica dos músculos avaliados nesse estudo.

Utilizando uma metodologia similar à do protocolo do estudo supracitado, Lepers et al.(4) avaliaram os efeitos dos parâmetros neuromusculares após cinco horas de pedalada. Nove ciclistas treinados pedalaram em intensidade constante, referente a 55\% da potência aeróbia máxima, com cadência de pedalada livre. O protocolo consistiu em avaliar o torque dos músculos extensores da articulação do joelho durante cinco contrações voluntárias máximas (CVMs), realizadas a cada 60 minutos. Os sujeitos paravam de pedalar e eram imediatamente colocados no dinamômetro, com a posição da articulação do joelho fixada em $60^{\circ}$.

Os resultados do pico de torque e do valor RMS dos músculos VL e VM, comparados com o pré-exercício, apresentaram redução signifi- 
cativa nos cinco momentos avaliados ( $\mathrm{H1}$ : - $-9 \%, \mathrm{H} 2:-8 \%, \mathrm{H3}:-10 \%, \mathrm{H} 4$ : $-16 \%, \mathrm{H} 5$ : $-18 \%$ e H1: $-16 \%, \mathrm{H} 2:-13 \%, \mathrm{H3}:-12 \%, \mathrm{H} 4:-14 \%, \mathrm{H} 5:-14 \%$, respectivamente). A partir dos resultados, ficou evidenciado que após cinco horas de exercício em intensidade moderada os músculos do quadríceps apresentaram indícios de fadiga.

Apesar de a carga ter sido mantida constante ao longo do teste, o que assegurou aos autores o controle dessa variável, teria sido interessante controlar $\mathrm{O} \mathrm{VO}_{2}$, principalmente a taxa de troca respiratória (RER), que é a razão entre a produção de dióxido de carbono $\left(\mathrm{VCO}_{2}\right)$ e o consumo de oxigênio. Essa taxa determina a predominância de qual sistema energético está sendo utilizado pelo atleta; valores abaixo de 1 indicam maior contribuição do sistema aeróbio e valores acima de 1, indicam maior contribuição do sistema anaeróbio(45-47).

À medida que o RER se aproxima de 1, a produção de ácido lático aumenta. Em seguida, ocorre a dissociação do mesmo em lactato e íon $\mathrm{H}^{+}$, afetando o acoplamento excitação-contração, as propriedades da membrana muscular e a propagação do potencial de ação. Essa informação ao longo do teste poderia ajudar a identificar as possíveis contribuições das demandas fisiológicas no processo de fadiga.

Moritani et al. ${ }^{(48)}$ determinaram a potência máxima no limiar de fadiga neuromuscular a partir da eletromiografia de músculos representativos do movimento da pedalada. O protocolo proposto utilizou, além da eletromiografia de superfície, a eletromiografia de profundidade para a análise do pico de ativação de unidades motoras (UMs) e análise de gases para determinação do limiar ventilatório (LV). Oito homens e 12 mulheres foram voluntários para esse estudo. Os sujeitos pedalaram em quatro diferentes cargas: 275, 300, 350 e 400W para os homens; e 200, 250, 300 e 350W para as mulheres.

Os testes foram randomizados e realizados no mesmo dia. Um intervalo de aproximadamente 25 minutos foi mantido entre cada carga avaliada para evitar a fadiga. A máxima duração de cada teste foi estipulada em dois minutos para todos os sujeitos. De acordo com os autores, os sujeitos apresentaram aumento na amplitude do sinal EMG e redução na MDF em todas as cargas avaliadas, provavelmente pelo progressivo aumento no número de UMs recrutadas e/ou aumento da freqüência de disparo para compensar o déficit das UMs fadigadas. A redução na MDF provavelmente está relacionada com a redução na velocidade de condução dos potenciais de ação nas fibras musculares daquelas unidades motoras fatigadas ${ }^{(49)}$.

Três sujeitos desse estudo foram voluntários para o uso da eletromiografia de profundidade. O objetivo foi analisar a atividade elétrica (amplitude) das UMs durante os testes com carga constante. Segundo os autores, UMs maiores, que produzem mais força e necessitam maior limiar de recrutamento, apresentam maior amplitude quando compradas com UMs menores. Os resultados apontaram aumento significativo no número de UMs recrutadas e na amplitude do sinal em cada uma das cargas avaliadas.

Bini et al. ${ }^{(50)}$ avaliaram a atividade elétrica de cinco músculos do membro inferior $(\mathrm{VL}$, rectus femoris - $\mathrm{RF}$, bíceps femoris - BF, tibialis anterior - TA e gastrocnemius medialis - GM) de oito triatletas experientes durante um teste simulado de $40 \mathrm{~km}$ contra-relógio. A cadência de pedalada utilizada durante o teste foi de livre escolha do atleta (preferida).

Paralelo à atividade elétrica dos músculos, foram também registrados $\mathrm{VO}_{2}$, RER, potência $\left(P_{0}\right)$, velocidade e cadência de pedalada nos quilômetros: 3, 10, 20 e 38 do teste. Os resultados apresentaram aumento significativo para as variáveis $\mathrm{VO}_{2}, \mathrm{P}_{\mathrm{O}}$, velocidade e valor RMS do $V L$ entre o início e o fim do teste (3km e $38 \mathrm{~km}$, respectivamente). Os autores atribuem essas mudanças à estratégia adotada pelos triatletas de realizar o teste de forma progressiva e mantendo a mesma cadência de pedalada.

Acredita-se que o aumento da atividade elétrica do VL esteja associado às forças aplicadas no pedal, principalmente à força normal; pois, segundo Gregoire et al.(51), os músculos monoarticulares geram força, enquanto os biarticulares são responsáveis pela transferência dessa através das articulações que cruzam.

Duc et al. ${ }^{(15)}$ avaliaram a atividade elétrica do VL, RF, BF e GM de nove experientes ciclistas durante um teste contra-relógio de $30 \mathrm{mi}$ nutos. Os dados de torque e EMG foram gravados a cada cinco minutos do teste. Os valores RMS e a MDF dos quatro músculos foram normalizados pelos valores referentes ao quinto minuto do teste. A atividade elétrica dos músculos flexores do joelho (BF e GM) apresentou tendência a aumentar ao longo dos 30 minutos, mas os resultados não foram significativos para os quatro músculos avaliados. Os resultados referentes à MDF apresentaram comportamento similar; no entanto, aumento significativo foi observado na MDF entre o quinto e o $10^{\circ}$ minuto.

A partir das informações do torque ao longo do teste, os autores calcularam a razão entre a atividade elétrica e o torque (EMG/torque) dos músculos avaliados. Os resultados de razão não indicaram mudanças significativas. Vale ressaltar que o aumento na razão EMG/ torque associada a torque constante indica fadiga periférica, e que razão EMG/torque constante associada a redução de torque indica fadiga central ${ }^{(14)}$. Os autores sugerem que a duração do teste não foi suficiente para causar alterações na ativação e na razão EMG/torque, pois a amostra do estudo foi composta por ciclistas altamente treinados, capazes de sustentar altas intensidades de esforço por longos períodos.

Hautier et al.(14) avaliaram a razão EMG/torque de dez ciclistas com o objetivo de observar as perdas de força e potência e o comportamento da ativação elétrica de cinco músculos do membro inferior (GM, RF, VL, BF e gluteus maximus - GL) durante testes de velocidade (sprints). Os sujeitos realizaram 15 repetições de cinco segundos de duração, com 25 segundos de intervalo, de sprint em um cicloergômetro equipado com um sensor de força (tensão) e um sensor óptico para o registro da velocidade e deslocamento. Os dados mecânicos e a atividade elétrica foram registrados na primeira e na $13^{3}$ repetição de cada indivíduo.

A média da potência máxima e o torque reduziram-se significantemente da primeira $(957,1 \pm 217,3 \mathrm{~W} ; 65,8 \pm 13,3 \mathrm{~N} \cdot \mathrm{m})$ para a $13^{\mathrm{a}}$ repetição $(849,3 \pm 199,3 W ; 61,8 \pm 12 \mathrm{~N} \cdot \mathrm{m})$. Com relação ao sinal EMG, foi observada diferença no valor RMS apenas no BF $(213 \pm 93 \mu \mathrm{V}-185$ $\pm 93 \mu \mathrm{V})$ e no $\mathrm{GL}(221 \pm 66 \mu \mathrm{V}-183 \pm 51 \mu \mathrm{V})$. Foi observado aumento na razão EMG/torque em detrimento da queda da força produzida pelos músculos sem mudança na atividade elétrica. Os autores atribuem a ocorrência de fadiga periférica a deficiência na capacidade de geração de força dos músculos, provavelmente decorrente de falha no acoplamento excitação-contração e falha na transmissão neuromuscular.

Ao avaliar o comportamento da potência máxima e da ativação elétrica dos músculos VL e BF de 12 ciclistas, durante dez sprints, com seis segundos de duração, Billaut et al. ${ }^{(52)}$ observaram queda de aproximadamente $11 \%$ no valor de pico da potência máxima (primeiro vs. $10^{\circ}$ sprint); no entanto, não houve diferença na integral do sinal eletromiográfico (iEMG). 
Em média, os sujeitos realizavam entre quatro e seis ciclos completos de pedalada durante cada sprint. Não foi observada diferença no período de ativação total do VL e BF entre o primeiro e o $10^{\circ}$ sprint. Em contrapartida, a análise do período de ativação dentro do sprint do segundo (CP2), terceiro (CP3) e quarto (CP4) ciclo de pedalada apresentou diferenças para o VL (CP2: 0,70 \pm 0,22s; CP3: 0,54 $\pm 0,20$ s; CP4: 0,43 $\pm 0,07$ s sprint $1 ; C P 2: 0,68 \pm 0,21 \mathrm{~s} ; \mathrm{CP} 3: 0,52 \pm 0,17 \mathrm{~s} ; \mathrm{CP} 4: 0,41$ $\pm 0,12$ s sprint 10$)$ e para o BF (CP2: 0,76 $\pm 0,25 \mathrm{~s} ; \mathrm{CP} 3: 0,47 \pm 0,16 \mathrm{~s} ; \mathrm{CP} 4$ : $0,41 \pm 0,14 \mathrm{~s}$ sprint $1 ;$ CP2: $0,78 \pm 0,17 \mathrm{~s} ; \mathrm{CP} 3: 0,47 \pm 0,15 \mathrm{~s} ; \mathrm{CP} 4: 0,44 \pm$ 0,11 s sprint 10), indicando alteração no padrão de coordenação dos músculos após a fadiga.

Em uma simulação de contra-relógio na distância de 4.000 metros (meio-fundo), Hetting et al.(53) observaram alterações na iEMG dos músculos VL e BF de oito ciclistas de elite. Os atletas foram submetidos a um protocolo que consistia de cinco testes de 4.000 metros com 48 horas de intervalo. No primeiro dia foi realizado um teste incremental para determinação do $\mathrm{VO}_{2 \mathrm{MAX}}$ e a $\mathrm{P}_{\mathrm{O}}$. O segundo dia foi destinado a um teste de 4.000 metros no menor tempo possível para determinar a potência de referência para os três testes subseqüentes: 95, 100 e $105 \%$ desse valor.

Os três testes de contra-relógio finais foram randomizados, e os sujeitos instruídos a pedalar os primeiros 2.000 metros na potência correspondente e a segunda metade no menor tempo possível. 0 sinal EMG dos músculos RF, VL e BF foi normalizado pelas respectivas CVMs de extensores e flexores do joelho. Os resultados apresentaram aumento significativo da iEMG para o VL e BF na segunda metade do protocolo, nas três intensidades avaliadas, enquanto que o RF não apresentou mudança no seu padrão de ativação.

Esses resultados demonstram que, independente da intensidade aplicada na primeira metade do teste, a alteração no padrão de ativação ocorreu em função da tentativa de realizar o teste no menor tempo possível, tornando evidente, assim, a instauração da fadiga periférica em detrimento da maior participação da via energética anaeróbia.

\section{Fadiga central}

O termo fadiga central pode ser definido como o conjunto de mecanismos que determinam a diminuição da contratibilidade muscular independente dos fatores intramusculares e/ou metabólicos. Segundo Ascensão et al. ${ }^{(54)}$, traduz-se em falha voluntária ou involuntária na condução do impulso nervoso que provoca redução do número de unidades motoras ativadas e diminuição da freqüência de disparo dos motoneurônios.

Os mecanismos centrais no processo da fadiga podem ser observados por meio de experimentos que utilizam a estimulação elétrica de músculos fadigados. De acordo com Bigland-Ritchie et al. ${ }^{(55)}$, a eletroestimulação permite avaliar o grau de comprometimento do SNC no processo de fadiga.

Lepers et al. ${ }^{(42)}$ utilizaram eletroestimulação durante um protocolo de contração voluntária máxima (CVM) com o objetivo de analisar os efeitos do ciclismo prolongado (duas horas) sobre a condução do potencial de ação (PA) dos músculos VL e VM. Os resultados apresentaram aumento significativo no tempo de condução do PA para o VL e o VM entre o início e o fim do teste $(8,6 \pm 1,3 \mathrm{~ms}$ para $10,7 \pm 2,7 \mathrm{~ms}$ e 7,8 \pm $3,2 \mathrm{~ms}$ para $8,7 \pm 2,7 \mathrm{~ms}$, respectivamente).

Além da diminuição na velocidade de condução do sinal, foi observada redução na amplitude e na área total do PA, indicando a presença de fadiga pré-sináptica e/ou fadiga na junção neuromuscular. Segundo os autores, as mudanças observadas no comportamento do PA podem estar associadas à redução na atividade da bomba sódio/potássio, mudanças metabólicas ( $\mathrm{H}^{+}$e fosfato inorgânico), e redução na liberação de cálcio $\left(\mathrm{Ca}^{2+}\right)$ pelo retículo sarcoplasmático.

Apesar da ampla utilização na análise dos prováveis fatores centrais da fadiga, os autores acreditam que a técnica da eletroestimulação recruta apenas as fibras que estão sob os eletrodos, limitando os resultados a pequena área do músculo. Além do mais, a capacidade de separar fadiga de ordem central de outras possíveis causas torna-se difícil, pois os mecanismos supra-espinhal e espinhal podem estar envolvidos.

Segundo Millet e Lepers(56), além dos fatores já descritos anteriormente e das prováveis alterações que ocorrem na excitabilidade do sarcolema, não está bem claro se o decréscimo na integral do sinal eletromiográfico (iEMG) observado depois de exercícios de longa duração pode ser completamente explicado pelas mudanças verifidadas na amplitude do PA, ou se realmente ocorreu fadiga de ordem central.

De acordo com os mesmos autores, quando o sinal eletromiográfico (EMG) durante uma CVM é utilizado para avaliar a existência de fadiga central, é necessário que o mesmo seja normalizado pela máxima amplitude do potencial de ação (M-wave). Normalmente, é utilizada a razão entre o valor root mean square (RMS) do sinal EMG e a M-wave (RMS/M-wave).

Uma grande diferença entre as mudanças no valor RMS e as na razão RMS/M-wave pode ser observada no processo de fadiga, demonstrando assim que o valor RMS ou mesmo o iEMG, durante uma CVM, não representam evidências de fadiga centra|(56).

Como foi observado, o declínio na capacidade de geração de força pode ocorrer em diferentes locais entre o SNC e os músculos, e métodos não-invasivos (i.e. eletromiografia de superfície, eletroestimulação, etc.) não são capazes de determinar com exatidão o local de origem da fadiga.

\section{CONCLUSÃO}

Apesar de a literatura específica acerca da manifestação do processo de fadiga durante o ciclismo envolvendo a aplicação de forças no pedal e a atividade elétrica dos músculos do membro inferior ser pequena, os estudos revisados apontam para incapacidade da manutenção da força desejada, como observado em outras tarefas motoras. No ciclismo, a fadiga tem sido relacionada com a perda da técnica de pedalada e quantificada por meio das mudanças nos padrões de ativação elétrica muscular. A partir do comportamento das forças aplicadas no pedal (magnitude e direção), é possível observar quais são as estratégias adotadas pelos ciclistas para minimizar os efeitos da fadiga. Embora a análise do sinal eletromiográfico forneça informações sobre os parâmetros neuromusculares, torna-se difícil determinar se o mecanismo de fadiga ocorre em detrimento de fatores centrais ou periféricos. O entendimento dos efeitos da fadiga sobre as forças aplicadas no pedal e sobre a atividade elétrica dos músculos do membro inferior em um protocolo que simule uma situação de competição específica possibilitaria traçar estratégias que minimizem as conseqüências do processo de fadiga.

Todos os autores declararam não haver qualquer potencial conflito de interesses referente a este artigo. 
REFERÊNCIAS BIBLIOGRÁFICAS

1. Burke ER. Physiology of cycling. In: Garret \& Kirkendall. Exercise and sport science. Philadelphia: Lippincott Williams \& Wilkins, 2000;759-70.

2. Abbis CR, Laursen PB. Models to explain fatigue during prolonged endurance cycling. Sports Med 2005;35:865-98

3. Diefenthaeler F, Candotti CT, Ribeiro J, Oliveira AR. Comparação de respostas fisiológicas absolutas e relativas entre ciclistas e triatletas. Rev Bras Med Esporte 2007;13:205-8.

4. Lepers R, Maffiuletti NA, Rochete L, Brugniax J, Millet GY. Neuromuscular fatigue during a long-duration cycling exercise. J Appl Physiol 2002;92:1487-93.

5. Hunter AM, Gibson SC, Lambert MI, Nobbs L, Noakes TD. Effects of supramaximal exercise on the eletromyographic signal. Britsh J Sports Med 2003;37:296-9.

6. Millet GY, Millet GP, Lattier G, Maffiuletti NA, Candau R. Alterations of neuromuscular function after a prolonged road cycling race. Int J Sports Med 2003;24:190-4.

7. Rossi L, Tirapegui J. Aminoácidos: bases atuais para sua suplementação na atividade física. Rev Bras Ciências Farm 2000;36:37-51.

8. Dimitrova NA, Dimitrov GV. Interpretation of EMG changes with fatigue: facts, pitfalls, and fallacies. J Electromyogr Kinesiol 2003;13:13-36.

9. Noakes TD. Physiological models to understand exercise fatigue and the adaptations that predict or enhance athletic performance. Scand J Med Sci Sports 2000;10:123-45.

10. Lees SJ, Franks PD, Spangenburg EE, Williams JH. Glycogen and glycogen phosphorylase associated with sarcoplasmic reticulum: effects of fatiguing activity. J Appl Physiol 2001;91:1638-44.

11. Fitts H. Cellular mechanisms of muscle fatigue. Physiol Rev 1994;74:49-94.

12. Noakes A, St Clair Gibson A. Logical limitations to the "catastrophe" models of fatigue during exercise in humans. Br J Sports Med 2004;38:648-9.

13. Coyle EF, Coggan AR, Hopper MK, Walters TJ. Determinations of endurance in well-trained cyclists. Appl Physiol 1988;64:2622-30

14. Hautier CA, Arsac LM, Deghdegh K, Souquet J, Belli A, Lacour JR. Influence of fatigue on EMG/Force ratio and cocontraction in cycling. Med Sci Sports Exerc 2000;32:839-43.

15. Duc S, Betik AC, Grappe F. EMG activity does not change during a time trial in competitive cyclists, Int J Sports Med 2005;26:145-50.

16. Bigland-Ritchie B, Jones DA, Hosking GP, Edwards RHT. Central and peripheral fatique in sustained maximum contractions of human quadriceps muscle. Clin Sci Mol Med 1978;54:609-14.

17. Enoka RM. Bases neuromecânicas da cinesiologia. São Paulo: Manole, 2000.

18. Sforzo GA, Touey PR. Manipulating exercise order affects muscular performance during a resistance exercise training session. J Strength Cond Res 1996;10:20-4.

19. Sharp A. Bicycles and tricycles. Cambridge, MA: MIT Press, 1977 (original publicado em 1896).

20. Hull ML, Davis RR. Measurement of pedal loading in bicycling - I. Instrumentation. J Biomech 1981;14:843-55

21. Rowe T, Hull ML, Wang EL. A pedal dynamometer for off-road bicycling. J Biomech Eng 1998;120:160-4.

22. Boyd TF, Neptune RR, Hull ML. Pedal and knee using a multi-degree-of-freedom pedal platform in cycling. J Biomech 1997;30:505-11

23. Candotti CT, Ribeiro J, Soares DP, Oliveira AR, Loss JF, Guimarães ACS. Effective force and economy of triathletes and cyclists. Sport Biomech 2007;6:32-43.

24. Gregor RJ, Broker JP, Rayan MM. The biomechanics of cycling. Exerc Sport Sci Rev 1991;19:127-69.

25. Burke ER. High-tech cycling. Colorado Springs: Human Kinetics, 1996.

26. Gregor RJ. Biomechanics of cycling. In: Garret \& Kirkendall. Exercise and Sport Science. Philadelphia: Lippincott Williams \& Wilkins, 2000;515-37.

27. Diefenthaeler F, Bini RR, Nabinger E, Laitano OL, Carpes FP, Mota CB, et al. Proposta metodológica para a avaliação da técnica da pedalada de ciclistas: estudo de caso. Rev Bras Med Esporte. 2008;14:155-8.

28. LaFortune MA, Cavanagh PR. Effectiveness and efficiency during bicycle riding. In: Matsui \& Kobayashi, editores. Biomechanics VIII-B. Champaign: Human Kinetics Publishers, 1983;928-36.

29. Too D. Biomechanics of cycling and factors affecting performance. Sports Med 1990;10:286-303.
30. Patterson RP, Moreno MJ. Bicycling pedaling forces as a function of pedaling rate and power output. Med Sci Sports Exerc 1990;22:512-6.

31. Amoroso A, Sanderson DJ, Henning EM. Kinematic and kinetic changes in cycling resulting from fatigue. Proceedings of 14th International Congress of Biomechanics. Paris. 1993;94-5.

32. Sanderson DJ, Black A. The effect of prolonged cycling on pedal forces. J Sports Sci 2003;21:191-9.

33. Carpes FP, Bini RR, Nabinger E, Diefenthaeler F, Mota CB, Guimarães ACS. Aplicação de força no pedal em prova de ciclismo $40 \mathrm{~km}$ contra-relógio simulada: estudo preliminar. Rev Bras Educ Fís Esp 2005;19:105-13.

34. Carpes FP, Rossato M, Faria IE, Mota CB. Bilateral pedaling asymmetry during 40-km cycling time-trial. J Sport Med Phys Fitness 2007;47:51-7.

35. Moritani T, Muro M, Nagata A. Intramuscular and surface electromyogram changes during muscle fatigue. J Appl Physiol 1986;60:1179-85.

36. Faria IE. Energy expenditure, aerodynamics and medical problems in cycling. Sports Med 1992;14:43-63.

37. De Luca CJ. The use of surface electromyography in biomechanics. J Appli Biomech 1997;13:135-63.

38. Li L, Caldwell GE. Muscle coordination in cycling: effect of surface and posture. J Appl Physiol 1998;85:927-34.

39. Baum BS, Li L. Lower extremity activities during cycling are influenced by load and frequency. J Eletromyogr Kinesiol 2003;13:181-90.

40. Diefenthaeler F, Bini RR, Laitano OL, Guimarães ACS, Nabinger E, Carpes FP, et al. Assessment of the effects of saddle on cyclists' pedaling technique. Med Sci Sports Exerc 2005;38(supplement):181.

41. Vøllestad NK. Measurement of human muscle fatigue. J Neurosci Methods 1997;74:219-27.

42. Lepers R, Hausswirth C, Maffiuletti NA, Brisswalter J, Hoecke JV. Evidence of neuromuscular fatigue after prolonged cycling exercise. Med Sci Sports Exerc 2000;32:1880-6.

43. Candotti CT, Castro FAS, Scheeren EM, Pressi AMS, Loss JF, Vaz MA, et al. EMG signal behavior in human vastus lateralis, tibialis anterior and soleus muscles during fatigue. Braz J Biomech 2004;9:15-9.

44. Herzog W, Zhang Y, Vaz MA, Guimarães ACS, Janssen C. Assessment of muscular fatigue using vibromyography. Muscle \& Nerve 1994;17:1156-61.

45. McLellan TM, Gass GC. Metabolic and cardiorespiratory responses relative to the anaerobic threshold. Med Sci Sports Exerc 1989;21:191-8.

46. Figueira TR, Denadai BS. Relações entre limiar anaeróbio, limiar anaeróbio individual e máxima fase estável de lactato em ciclistas. Rev Bras Cien Mov 2004;12:91-5.

47. Moseley L, Jeukendrup AE. The reliability of cycling efficiency. Med Sci Sports Exerc 2001;33:621-7.

48. Moritani T, Takaishi T, Matsumoto T. Determination of maximal power output at neuromuscular fatigue threshold. J Appl Physiol 1993;74:1729-34.

49. Kupa EJ, Roy SH, Kandarian SC, De Luca CJ. Effects of muscle fiber type and size on EMG median frequency and conduction velocity. J Appl Physiol 1995;79:23-32.

50. Bini RR, Carpes FP, Diefenthaeler F, Mota CB, Guimarães ACS. Physiological and electromyographic responses during 40-km cycling time trial: Relationship to muscle coordination and performance. Sci Med Sport. 2008;11:363-70.

51. Gregoire L, Veeger HE, Huijing PA, Van Ingen Schenau GJ. Role of mono and biarticular muscles in explosive movements. Int J Sports Med 1984;5:301-5.

52. Billaut F, Basset FA, Falgairette G. Muscle coordination changes during intermittent cycling sprints. Neurosci Lett 2005;380:265-9.

53. Hetting FJ, De Koning JJ, Broersen FT, Geffen PV, Foster C. Pacing strategy and the occurrence of fatigue in 4000-m cycling time trials. Med Scie Sports Excer 2006;38:1484-91.

54. Ascensão A, Magalhães J, Oliveira J, Duarte J, Soares. Fisiologia da fadiga muscular. Delimitação conceptual, modelos de estudo e mecanismos de fadiga de origem central e periférica. Rev Port Cien Desp 2003;3:108-23.

55. Bigland-Ritchie B, Furbush F, Woods J. Fatigue of intermittent submaximal voluntary contractions: central and peripheral factors. J Appl Physiol 1986;61:421-9.

56. Millet $G Y$, Lepers R. Alterations of neuromuscular function after prolonged running, cycling and skiing exercises. Sports Med 2004;34:105-16. 